\title{
Contrôle de la pureté des semences de base et de prébase mâle-stériles cytoplasmiques par hybridation moléculaire avec un plasmide mitochondrial
}

\author{
A Bervillé, P Faivre-Rampant, S Santoni, E Moreau \\ INRA Dijon, station de génétique et amélioration des plantes, BV 1540, 21034 Dijon Cedex, France
}

(Reçu le 5 mars 1989; accepté le 11 septembre 1990)

\begin{abstract}
Résumé - Pour produire des semences hybrides, la lignée mâle-stérile cytoplasmique ne doit pas être contaminée par le mainteneur. Si tel est le cas, l'hybride contiendra des plantes hétérogènes, avec pour conséquence, une diminution du rendement. Pour identifier spécifiquement le cytoplasme du mainteneur, nous proposons d'utiliser une méthode d'analyse moléculaire. Un plasmide mitochondrial spécifique des mitochondries du mainteneur est utilisé comme sonde moléculaire pour révéler la présence éventuelle de séquences du plasmide dans le lot de semences mâle-stériles cytoplasmiques. La méthode est simple à manipuler, d'un coût raisonnable, très rapide et plus précise que les contrôles au champ. En routine, nous contrôlons 1000 graines sèches de tournesol. En divisant le lot en 6 parties, nous dénombrons les graines contaminantes du mainteneur. Pour la betterave, nous avons divisé le lot de 1 000 graines en 67 parties, ainsi nous déterminons avec précision le nombre de graines contaminantes du mainteneur.
\end{abstract}

semence / minicercle / stérilité mâle cytoplasmique / tournesol / betterave

Summary - Control of cytoplasmic male-sterile seed stocks by molecular hybridization with a mitochondrial plasmid. To produce hybrid seeds, the cytoplasmic male sterile line must be free of contamination by maintainer seeds. The hybrid will then contain heterogeneous plants which will decrease the yield. To specifically identify the maintainer cytoplasm, we propose the use of a molecular method. A mitochondrial plasmid, specific to the maintainer cytoplasm is used as a molecular probe to reveal the possible presence of plasmid sequences in the CMS seed stocks. The method appears to be simple, at a reasonable cost, very fast and more accurate than field controls. For sugar beet, we first determined the level of the minicircle $c$ in one seed by comparison with the probe loaded as a ladder between $5 \mathrm{pg}$ to $1000 \mathrm{pg}$, (fig .1). Because of a slight homology between minicircle c (130 pg per seed) and the minicircle a, the hybridization signal of dot blot does not allow 1 maintainer seed to be identified in less than 10 CMS seeds, (fig 2). In order to conciliate a deep hybridization signal and a low number of sub-samples, we divided the 1000 seed samples into 67 sub-samples thus allowing us to accurately determine the number of contaminated maintainer seeds. We routinely checked 1000 dry seeds for sunflower (table I). The sample in an assay was compared to controls artificially contaminated (fig 3). The method appeared to be expensive and relatively uncertain when CMS controls were also contaminated with maintainer seeds. By dividing the stock into 6 sub-samples, we were able to determine the maintainer contaminant seeds within each sub-sample (fig 4). We applied this method to 20 commercial samples from various companies (table II). The results of the molecular control fit the controls in the field.

seed / minicircle / cytoplasmic male sterility / sunflower / sugarbeet

\section{INTRODUCTION}

Pour produire des semences hybrides à une échelle commerciale, la méthode la plus efficace consiste à castrer génétiquement la lignée utilisée comme parent femelle de l'hybride. Pour cette raison, une stérilité mâle cytoplasmique (SMC) est préférée, quand elle est disponible, puisque après croisement par un mainteneur, la descendance sera homogène mâle-stérile. Tou- tefois, pour produire l'hybride, il est nécessaire que le parent mâle apporte des allèles dominants restaurant la fertilité mâle de l'hybride : ce parent mâle est dit restaurateur (Vear, 1988; Bervillé, 1988). L'utilisation d'une stérilité mâle cytoplasmique impose donc la manipulation du mainteneur de stérilité pour multiplier la forme mâlestérile. Cette opération doit être parfaitement contrôlée afin d'éviter la contamination des semences du lot à SMC par celles du mainteneur. 
Si une telle contamination se produit, l'avantage de multiplication du mâle-fertile en autofécondation acroîtra la proportion de plantes mâlefertiles à chaque génération, le mâle-stérile sera donc rapidement inutilisable. II sera alors nécessaire d'épurer le champ de production de semences de toutes les plantes mâle-fertiles afin de ne récolter que des graines sur les plantes à SMC.

Ainsi est-il important pour les sélectionneurs de déterminer le taux de plantes mâle-fertiles dans les lots de semences de prébase et de base mâle-stériles. Si la production de l'hybride impose d'utiliser plusieurs lignées femelles en mélange ou un hybride à SMC, le contrôle devra être réalisé pour chaque lignée à SMC. La seule méthode efficace consiste à cultiver quelques centaines, voire un millier, de plantes du lot à contrôler et ce jusqu'à la floraison. Les plantes seront examinées pour leur fertilité mâle mais on déterminera aussi si elles correspondent ou non au type de la lignée. La culture des plantes pour les contrôles est réalisée généralement à contre saison dans l'hémisphère sud ou en serre.

En aval, l'utilisation d'une stérilité mâle cytoplasmique pour produire des semences hybrides de tournesol entraîne des risques d'impuretés que les sélectionneurs regroupent en 2 classes.

Les impuretés de classe I représentent des plantes vigoureuses mais ne correspondent pas au standard de la variété : elles sont très certainement dues à la fécondation de plantes à SMC par un pollen ne provenant pas du parent mâle. Elles seront, soit mâle-fertiles, soit mâle-stériles selon l'allèle de restauration dominant ou récessif apporté par le pollen étranger. Quoiqu'il en soit, le cytoplasme de ces plantes sera du même type que celui du parent femelle, c'est-à-dire du type mâle-stérile.

En ce qui concerne les impuretés de classe II, les plantes n'expriment pas de vigueur hybride. Elles proviennent du croisement par un mainteneur, et étant donné leur manque de vigueur, c'est vraisemblablement du pollen du propre mainteneur de la lignée qui a servi de parent mâle. Leur cytoplasme doit donc être de type mâle-stérile. Quand les impurerés de classe II sont mâle-fertiles et d'un niveau de vigueur équivalent à la lignée, elles sont très certainement dues à des graines de mainteneur qui polluent le lot de semences à SMC.

Pour déterminer qu'une plante est mainteneuse de stérilité mâle, il faut réaliser le croisement avec un mâle-stérile (test-cross) et exami- ner la descendance. Elle sera homogène mâlestérile si la plante est mainteneuse de stérilité mâle pour le cytoplasme porté par le mâlestérile.

Il est donc économiquement très lourd et irréaliste de vérifier le cytoplasme des contaminants des lots de semences de base. Pourtant, pour diminuer leur taux et améliorer la production de semences hybrides, il faut en déterminer l'origine. Le sélectionneur qui reçoit les lots de semences de plusieurs producteurs, pour bien faire, devrait attendre les résultats des contrôles avant de les traiter en mélange. Ceci lui poserait des problèmes de stockage et de conditionnement onéreux. Toutefois, si un lot de production est fortement contaminé, l'ensemble après mélange sera pollué.

Le domaine de la biologie moléculaire permet d'élaborer une méthode d'identification sûre du mainteneur de la SMC ainsi que de la quantification de la pollution du lot de semences de base à SMC par le mainteneur.

Les semences du stock à SMC sont obtenues par croisement des plantes à SMC avec les plantes mainteneuses de stérilité mâle. Les 2 lignées ne diffèrent que par leur cytoplasme; elles sont isogéniques pour tous les caractères nucléaires.

Le caractère de stérilité mâle cytoplasmique est codé dans l'ADN mitochondrial par le chromosome maître. En plus de ce chromosome, il existe chez la betterave, le tournesol, le maïs (Hansen et Marcker, 1984; Pérez et al, 1988; Kemble et Bedbrook, 1980), des molécules beaucoup plus petites appelées plasmid-like; elles ont une replication autonome. Pour les formes à SMC et mainteneuses de la plupart des espèces, notamment du tournesol et de la betterave, les formes plasmidiques sont différentes, ce qui permet de reconnaître les cytoplasmes après électrophorèse de I'ADN mitochondrial natif. La reconnaissance des cytoplasmes est encore plus évidente et sûre si le plasmide existant chez le mainteneur est totalement absent chez la plante à SMC. Cette situation est décrite chez le tournesol avec la stérilité mâle d'origine française (Leclercq, 1969) sans plasmide alors que le cytoplasme du tournesol en possède un de 1415 paires de base. Ce plasmide (pIT) a été cloné et entièrement séquencé (Pérez et al, 1988); il est utilisé pour identifier avec certitude le cytoplasme des mainteneurs par hybridation moléculaire. Chez la betterave, une situation semblable existe pour les plantes à SMC utilisée 
(Owen, 1945). Les mainteneurs de $B$ vulgaris possèdent le plasmide $\mathrm{C}$ alors que la forme stérile en est dépourvue (Powling, 1981). Le plasmide c cloné est désigné par pO, il permet par hybridation moléculaire d'identifier avec certitude le cytoplasme du mainteneur des betteraves (Hansen et Marcker, 1984; Thomas, 1986).

Dans un article précédent (Faivre-Rampant et al, 1989), nous avons montré la faisabilité de la méthode en étudiant des lots de semences artificiellement contaminés par des graines de mainteneur. Pour cet article, nous avons utilisé la méthode pour des semences de base et de prébase fournies par plusieurs sociétés. Nous avons préparé les acides nucléiques (AN) de graines sèches correspondant à des lots de semences de betterave ou de tournesol. Nous avons déposé les AN sur une membrane de nylon, selon la technique de dépôt en tache (dot blot) pour les hybrider avec les plasmides, soit pO, soit pIT. Le signal d'hybridation permet, par rapport à des contrôles, de déterminer rapidement le taux de contamination du lot par les graines de mainteneur. Le coût du contrôle est tout à fait raisonnable, donc applicable de façon systématique sur les lots de producteurs, un tel contrôle peut éviter de mélanger des lots pollués à des lots exempts de contamination.

\section{MATÉRIEL ET MÉTHODES}

\section{Matériel végétal}

Le matériel végétal nous a été remis par les sociétés selon leur propre code. Nous l'avons renommé. $\mathrm{Ce}$ sont les génotypes utilisés actuellement dans la production de semences commerciales. Les échantillons de chaque lot correspondent à 1000 graines alors que les sélectionneurs ont l'habitude d'utiliser des lots de 200 à 1000 graines pour leurs propres contrôles. Nous supposerons que les lots sont homogènes pour les contaminants recherchés.

Pour la betterave, en prévision d'une analyse des lots de 1000 graines par 100,67 ou 50 parts, nous avons étudié des témoins de contamination qui correspondent à une part. Nous avons donc remplacé 0,1 , 2, 3 ou 4 graines des 10,15 ou 20 graines de plantes à SMC par celles du mainteneur. Pour le tournesol, nous avons essayé plusieurs méthodes pour traiter les 1000 graines :

- les 1000 graines sont broyées ensemble. Les témoins utilisés sont des lots purs à SMC (forme $A$ des sélectionneurs), contaminés par $0,1,2,3,5$ ou 10 graines du mainteneur (forme B des sélectionneurs);
- les 1000 graines sont divisées en 6 parts de 166 et 167 graines broyées séparément. Les témoins sont préparés en ajoutant à 166 graines de plantes à SMC, 0,1 ou 2 graines du mainteneur.

\section{Méthodes de préparation et d'analyse}

Les méthodes de préparation des acides nucléiques à partir des graines sèches sont dérivées de la méthode décrite par Faivre-Rampant et al (1989) et Santoni et al (1991). Les méthodes d'électrophorèse, de préparation des filtres pour les hybridations, ainsi que les hybridations sont celles de Maniatis et al (1982).

Les hybridations ont été réalisées avec les inserts purifiés des plasmides bactériens vecteurs.

\section{RÉSULTATS ET DISCUSSION}

La méthode de préparation de l'ADN des graines sèches que nous utilisons permet d'obtenir de l'ADN hydrolysable par les enzymes de restriction. Cet ADN est cependant contaminé par de l'ARN dégradé. Nous avons donc dénommé la fraction ADN fraction acides nucléiques (= AN).

\section{Betterave}

Tous les mainteneurs de la stérilité mâle source Owen portent le plasmide $c$ alors que les plantes à SMC Owen en sont dépourvues. Toutefois, les mainteneurs portent un plasmide a qui contient une séquence (environ 100 paires de base (bp)) homologue au plasmide c (Thomas, 1986). La sonde $\mathrm{pO}$ s'hybride donc faiblement au plasmide a; il s'ensuit un faible signal en dot blot qui correspond à environ 1/50 d'une graine mainteneur

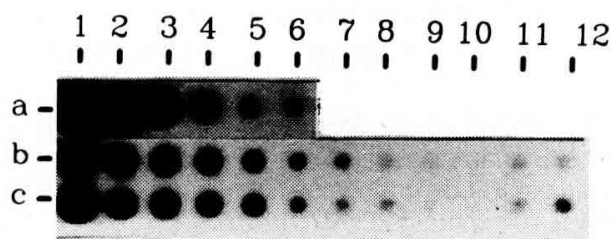

Fig 1. Evaluation de la quantité du minicercle $c$ dans les graines de mainteneur de la betterave à sucre. Signaux de dépôts en tache d'acides nucléiques, préparés a partir de graines sèches, hybridés par le plasmide pO $\left(0,5 \times 10^{9}\right.$ dpm $\mu g-1$ (ADN). Ligne a = dépót de l'ADN en pg du plasmide pO; colonne 1: 1 000; colonne 2 : 500; colonne 3 : 100; colonne $4: 50$; colonne $5: 10$; colonne $6: 5$; lignes b et $\mathrm{C}=$ dépôt des $\mathrm{AN}$ totaux de 2 graines du mainteneur (M) puis dilutions successives par $2,5,10,20,50,100,200,500$ et 1000 ; colonnes 11 et 12 , AN totaux de 2 graines a SMC. 
pour une graine à SMC (fig 1). C'est pour cette raison que nous avons fractionné les 1000 graines en parts. La marge de détection du minicercle $c$ dans l'échantillon d'ADN est donc faible en routine. En admettant qu'il faut environ $5 \mathrm{pg}$ du mini cercle $\mathrm{c}$ pour que la détection soit sûre, la fraction de la graine mainteneur doit représenter au plus $1 / 20$. Nous avons utilisé des parts de 20 graines au plus et essayé 15 et 10 graines. La quantité totale d'ADN d'une graine a été estimée, par plusieurs dosages non montrés, en moyenne à $350 \mathrm{ng}$. En moyenne, la quantité du plasmide $c$ par graine mainteneur (M) est estimée à $130 \mathrm{pg}$ (fig 1, colonne 1). Nous avons donc comparé les signaux donnés par $0,1,2,3$ ou 4 graines du mainteneur et du SMC pour 10, 15 ou 20 graines au total. L'intensité des signaux augmente en fonction du nombre de graines du mainteneur, avec toutefois des variations (fig 2 lignes a). Elles sont dues, soit à la variation des quantités de plasmides par graine, soit au cœefficient d'extraction, variable d'un échantillon à l'autre. Nous avons étudié les signaux des mélanges des AN de graines $M$ et SMC obtenus séparément, pour reproduire les proportions des graines en mélange (fig 2, lignes b). La reproductibilité des signaux est meilleure; de plus, les variations d'intensité sont plus régulières, ce qui montre que le cœfficient d'extraction est la source de variation majeure des signaux. L'automatisation du broyage devrait améliorer la régularité du rendement. Avec des parts de 20 , nous

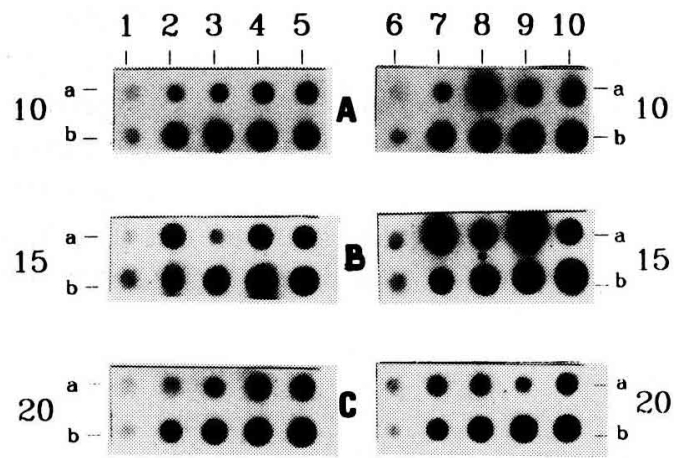

Fig 2. Comparaison du broyage des graines et du mélange des AN. Signaux de dépôts en tache d'acides nucléiques préparés à partir de graines sèches, hybridés par le plasmide pO $\left(0,5 \times 10^{9} \mathrm{dpm} \cdot \mu \mathrm{g}-1\right.$ (ADN). Colonnes 1 à 5 et répétition de 6 à $10: A=10$ graines broyées ensemble : ligne a colonnes 1 et $6: 0$ mainteneur $(M)+10$ SMC; colonnes 2 et 7 : $1 M+9$ SMC; colonnes 3 et $8: 2 M+8$ SMC; colonnes 4 et $9: 3 \mathrm{M}+7 \mathrm{MSC}$; colonnes 5 et $10: 4 M+6$ SMC; ligne $b=$ mélange d' AN préparés de graines à SMC et $M$ dans les mêmes proportions que ligne $a$. $B$ : ligne $a=15$ graines broyées ensemble, contaminées par $0,1,2,3$, ou $4 \mathrm{M}$; ligne $b=$ mélange d' AN dans les mêmes proportions. $C$ : ligne $a=$ 20 graines broyées ensemble contaminées par $0,1,2,3$ ou $4 \mathrm{M}$; ligne $\mathrm{b}=$ mélange d' $A N$ préparés de graines à SMC et $M$ dans les mêmes proportions que ligne a. réduisons le nombre de broyages (50) mais la détection d'une graine de mainteneur contaminante n'est pas nette (fig $2 \mathrm{C}$, ligne $\mathrm{b}$, colonne 2). En retenant l'effectif de 15 graines pour les parts, nous concilions une bonne détection du signal pour un contaminant, avec un nombre un peu réduit de broyages à effectuer : 67 au lieu de 100. En effet nous avons montré que le dénombrement des mainteneurs contaminants est possible puisque nous avons retrouvé dans un lot parfaitement mâle-stérile, les contaminants apportés (Faivre-Rampant et al, 1989). La présence d'une graine de mainteneur parmi 14 graines à SMC induit un signal qui ne peut se confondre avec celui des graines de plantes à SMC, car il est 10 fois plus fort (fig 2B). Au-delà d'une graine, la reproductibilité du signal n'est pas suffisante pour permettre en routine la quantification des graines du mainteneur d'une part (fig 2). Nous conviendrons donc que le signal positif d'une tache est donné par une graine mainteneuse contaminante. Si le lot est peu contaminé (taux de contamination < 1\%), la probabilité que 2 graines de mainteneur soient réunies dans une même part parmi 65 est 0,01 donc faible, d'après Lefort (1967).

Pour le dépôt en tache, nous avons choisi de déposer $1 / 4$ des AN totaux obtenus de 10,15 ou 20 graines, donc environ 3,4 ou 5 graines, soit respectivement 1, 1,3 et 1,6 $\mu \mathrm{g}$ d'AN. Le reste des AN est conservé congelé pour être réutilisé en cas de contestation du résultat.

L'analyse, par cette méthode, d'un lot à contrôler sera certes, fastidieuse, mais le dénombrement sans ambiguité des mainteneurs contaminants sera réalisable.

\section{Tournesol}

Tous les mainteneurs de stérilité mâle français portent le plasmide de $1415 \mathrm{bp}$, et éventuellement un deuxième plasmide dérivé du précédent par une délétion : pIC (Pérez et al, 1988).

Les AN extraits d'une graine unique du mainteneur représentent environ $20 \mu \mathrm{g}$. Une fraction de $0,2 \mu \mathrm{g}$ permet un signal d'hybridation alors que $20 \mu \mathrm{g}$ de l'extrait à SMC ne donneront pas de signal dans nos conditions d'hybridation, (non rapporté en détail dans cet article).

Nous avons quantifié le signal fourni par 0,1 , 5 et 10 graines du mainteneur dans 1000 graines à SMC. Pour cela, nous avons déposé les quantités croissantes de $2,10,20$ et $30 \mu \mathrm{g}$ d'AN 

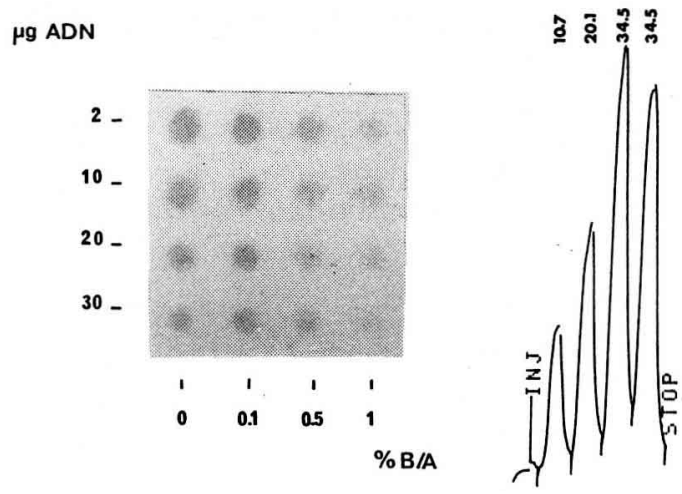

- as as 1

Fig 3. a. Signaux d'hybridation obtenus par dépôts en tache d'acides nucléiques préparés à partir de 1000 graines sèches de tournesol. Des dépôts de quantités croissantes de $2,10,20$ et $30 \mu \mathrm{g}$ d'AN pour 4 échantillons contenant : $0,1,5$ ou 10 graines du mainteneur (B) pour 1000 graines mâlestériles cytoplasmiques (A). La sonde plT avait une activité spécifique de $0,5 \times 10^{9} \mathrm{dpm}{ }^{2} \mathrm{~g}^{-1}$ (ADN). L'autoradiographie a été exposée $14 \mathrm{~h}$. b. Densitogramme des 4 dépôts correspondant à $10 \mu \mathrm{g}$ d'acides nucléiques.

des 1000 graines (fig 3). A côté sont déposées les mêmes quantités d'AN correspondant aux 4 lots témoins contaminés respectivement à $0,1,5$ et $10 \%$. Par analyse densitométrique, la contamination du lot à tester est, par rapport aux témoins, estimée à $0-1 \%, 1-5 \%, 5-10 \%$ ou $\geq$ $10 \%$ 。 respectivement (tableau I).

Cette méthodologie, bien que réalisable pour des contrôles, souffre de plusieurs inconvénients :

- le taux de contamination n'est qu'estimé;

- 4 témoins sont réalisés pour un lot à analyser; de plus pour chacun des témoins, la plage de comparaison valable dépend du nombre de contaminants. Ainsi, pour l'échantillon de la figure 3 entre $0,5 \%$ et $1 \%$, le signal ne progresse pas; mais le lot en essai correspondant n'étant contaminé qu'à moins de $0,5 \%$, c'est acceptable. Pour tous les lots en essai nous avons vérifié que les témoins étaient satisfaisants;

- Le coût élevé des semences de base nécessaires est élevé et certains témoins se sont révélés eux-mêmes contaminés. Les signaux ne sont plus progressifs; le lot à analyser ne peut être situé dans la gamme (non montré), la gamme artificiellement contaminée devient inutilisable.

Nous avons donc renoncé à cette méthode et fractionné le lot à analyser en 6 parts. Trois dépôts sont réalisés correspondant à : 2,10 et 20 $\mu \mathrm{g}$ d'AN. Les signaux sont comparés à ceux des témoins, 166 graines SMC, auxquelles sont ajoutées 0,1 ou 2 graines du mainteneur. La comparaison a été faite visuellement et au densitomètre. L'appréciation visuel est simple et suffisante.

Nous avons ainsi analysé 21 échantillons de 5 sociétés (tableau II). La comparaison des 6 taches (dots) d'une part révèle en fait une hétérogénéité des 6 parts. En comparant les 6 taches avec les témoins artificiellement contaminés, nous avons dénombré les contaminants (fig 4).

Quand le taux de contaminants du lot est élevé (>1\%), le dénombrement est impossible avec 6 parts; il faudrait augmenter le nombre de sous-parts. Toutefois le surcroît de travail ne se justifie pas car, avec 6 parts, un dénombrement jusqu'à 12 contaminants est réalisé. Au-delà le

Tableau I. Appréciation de la contamınation en graines du mainteneur dans les semences de base à SMC (pour 1000 graines) par quantification du signal des acides nucléques préparés de 1000 graines, hybridés par la sonde

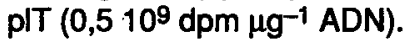

Observations des contaminants pour 1000 graines

Echantillons

\begin{tabular}{lll}
\hline 1 & $1<<5$ & $1<<5$ \\
2 & $>10$ & $>20$ \\
3 & 0 & $5<<10$ \\
4 & $(a)$ & $<1$ \\
5 & 0 & $1<5$ \\
6 & 0 & $1<<$ \\
7 & 0 & $<1$ \\
8 & 0 & $>10$ \\
\hline
\end{tabular}

(a) non déterminé du témoin

du lot à contrôler 
Tableau II. Taux de contamination (mainteneur/MSC), observé par le test moléculaire pour les échantillons traités en 6 parts; comparaison avec le taux des plantes mâle-fertiles observées au champ.

\section{Code échantillon}

Test moléculaire taux de contamination sur 1000 graines
Contaminants/effectif observé ou estimations au champ (a)

\begin{tabular}{lllll}
\hline G10276 & 3 & $0,3-11(\mathrm{~b})$ & $0 / 300$ & $0-7$ (b) \\
G10275 & 7 & $2-17$ & $2 / 300$ & $0-11$ \\
G7986 & 4 & $0,7-12,5$ & $1 / 203$ & $0-9$ \\
G7599 & 9 & $3,1-19,9$ & $1 / 138$ & $0-72$ \\
G7387 & 8 & $2,6-18,5$ & $1(a)$ & $0-7,4$ \\
G7388 & 5 & $1,1-14,1$ & $5,5(a)$ & $1,1-14,1$ \\
G7381 & $<2$ & $0,1-9,2$ & $1 / 898$ & $0-7,4$ \\
G7382 & 7 & $2-17$ & $4 / 718$ & $0,5-9$ \\
G7987 & 9 & $3,1-19,9$ & $1 / 242$ & $0-5,9$ \\
G7389 & 0 & $0-0,37$ & 0 & $0-0,37$ \\
G9706 & 2 & $0,1-9,2$ & $1 / 210$ & $0-6$ \\
G7386 & 3 & $0,3-11$ & $2.6(a)$ & $2-10$ \\
G7390 & 1 & $0-7,4$ & $1 / 200-1 / 2500$ & $0-6$ \\
G5000 & 6 & $1,5-15,6$ & $1.3(a)$ & $0-7,4$ \\
G5001 & 5 & $1,1-14,1$ & $1.8(a)$ & $0-7,4$ \\
G6001 & $>10$ & $3,7->21$ & $80(a)$ & $28-140$ \\
G6101 & 5 & $1,1-14,1$ & $21.7(a)$ & $11,3-33,6$ \\
G6201 & 5 & $1,1-14,1$ & $5(a)$ & $1,1-14,1$ \\
G3000 & 0 & $0-0,37$ & $0(a)$ & $0-0,37$ \\
G3001 & $10<<15$ & $21-30$ & $1,5 \%(a)$ & $18-30$ \\
& & & & \\
\hline
\end{tabular}

(a) effectif non déterminé, mais d'au moins 1000 plantes;

(b) intervalle de confiance à $99 \%$ pour le taux de contamination exprimé pour 1000 graines.

lot est suffisamment déprécié pour que le dénombrement précis des contaminants ne se justifie pas.

La plupart des sociétés ont contrôlé les mêmes lots de semences au champ en observant la floraison mâle et le phénotype des plantes anormales. Les sociétés ont contrôlé des effectifs assez variables : de 160 à 2500 plan-

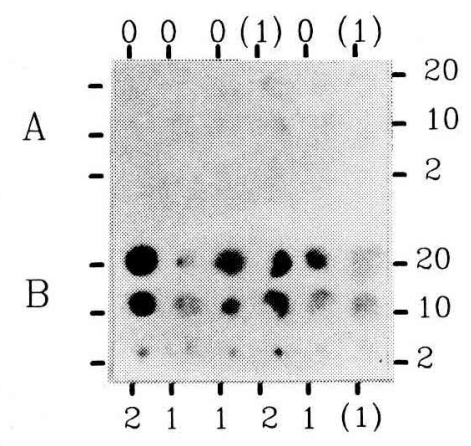

Fig 4. Signaux de dépôts en taches d'acides nucléiques hybridés avec la sonde pIT $\left(0,5 \times 10^{9} \mathrm{dpm} \mu \mathrm{g}^{-1}\right.$ (ADN)) après $24 \mathrm{~h}$ d'exposition. 20,10 et $2 \mu \mathrm{g}$ d'AN sont déposés. Les 6 colonnes correspondent aux 6 parts du lot de base en essai. A. Lot G7381. Les nombres au-dessus désignent les contaminants; () signifie douteux. B. Lot G7382. Les nombres audessous désignent les contaminants. tes. Les fluctuations statistiques pour les petits échantillons sont donc importantes, et dans tous les cas, les résultats des tests au champ sont compatibles avec ceux du contrôle moléculaire. Dans le tableau II, nous donnons les intervalles de confiance à $99 \%$ des valeurs d'après la table de la loi binômiale : tous les intervalles de confiance se recouvrent. Les 2 tests sont toujours compatibles.

\section{CONCLUSION}

Nous avons donc démontré que, pour la betterave et le tournesol, l'utilisation d'une sonde de plasmide mitochondrial spécifique du mainteneur est utilisable pour repérer les lots de semences de base à SMC contaminés par des mainteneurs. Les contaminants peuvent être dénombrés en divisant le lot de 1000 graines en 6 parts. Un plus grand nombre de parts ne se justifie pas pour le tournesol. En revanche pour la betterave, un grand nombre de parts est nécessaire car le plasmide pO n'est pas parfaitement 
spécifique du mainteneur. Nous avons éliminé du plasmide pO la séquence qui se retrouve dans l'autre plasmide minicercle a. L'amélioration de la spécificité du signal ne permet toutefois pas d'utiliser des parts de plus de 20 graines. L'obtention d'une sonde plus spécifique ne compense pas la faible quantité du minicercle $c$ dans l'échantillon à analyser. Le dénombrement des contaminants est précis. Pour l'instant, nous ne disposons pas d'un broyeur pour réaliser un nombre important de broyages sur les petits échantillons. Avec un broyeur adapté, le tournesol pourrait être traité comme la betterave pour accroitre la sensibilité du dénombrement des contaminants.

La méthode dispense d'utiliser des témoins artificiellement contaminés, puisque les parts extrêmes feront office de témoins internes, - non contaminées : pas de signal - contaminées : témoins de signaux apparents.

Le coût des contrôles moléculaires a été estimé à 700FF (matériel et main-d'œuvre). Les sélectionneurs estiment que le contrôle d'un lot revient 2 000-3 000FF en serre ou à contre-saison. Les semences de base et de prébase coûtent de 1-3FF la semence pour le SMC et beaucoup plus pour le mainteneur. II apparaît donc clair que le coût des lots témoins artificiellement contaminés grève le prix de revient du test. C'est la raison pour laquelle le sélectionneur accepte de perdre en précision sur le dénombrement en n'utilisant pas de témoin (fig 4).

La technique de détermination des mainteneurs contaminants dans les lots de semences à SMC par une sonde moléculaire s'est révélée avantageuse par rapport au contrôle des plantes à la floraison. La méthode moléculaire présente plusieurs avantages dont la rapidité, puisque entre le broyage des graines et l'obtention des résultats, seulement quelques j sont nécessaires. II faut plusieurs mois pour observer la floraison. La méthode moléculaire utilise des graines sèches, ce qui ne peut être qu'un avantage quand les graines fraîchement récoltées entrent en dormance, après séchage, pour quelques semaines.

La méthode moléculaire permet de traiter plus de graines que le contrôle au champ. Sa précision en est donc meilleure.

La méthode moléculaire n'utilise pas de moyens onéreux ni d'équipements sophistiqués. Seule l'hybridation par une sonde radioactive pose des problèmes de sécurité et nécessite un agrément. Toutes les étapes se situant en amont peuvent se réaliser dans n'importe quel labora- toire, ensuite les filtres pourront être envoyés pour être hybridés dans un laboratoire agréé. Nous avons récemment vérifié que le marquage froid du plasmide $\mathrm{pO}$ permettait de révéler le minicercle $\mathrm{c}$ des mainteneurs de betterave (Santoni et al, 1991).

La méthode présente beaucoup d'intérêt pour la recherche des séquences d'ADN ou d'ARN qui correspondent à des pathogènes ou à des génotypes contaminants. Les sondes adaptées sont souvent disponibles.

\section{REMERCIEMENTS}

Nous remercions les sociétés : Maribo France, KWS France, Van der Have France, SESCA, GFS, Deleplanque et cie, contrat $n^{\circ} 9157 \mathrm{~A}$ sur la betterave et le GIE Protournesol, le GIE AMSOL et le GNIS ainsi que Cargill, Caussade semences, Maïsadour, NorthrupKing, Pioneer-France, RAGT, Rhône-Poulenc Agrochimie, Rustica Bio-Recherches, Semundo Maïs, SDME, UCST, Van der Have France, contrat n`9190A sur le tournesol.

Nous remercions également $M$ Lund et $C$ Pérez pour les dons de plasmides (respectivement le pO et le plT) J Delbut et C Picard pour leur excellent travail technique.

\section{RÉFÉRENCES}

Bervillé A (1988) Quelques définitions et généralités. In: Cytoplasmic genetic variability and cytoplasmic male sterility (A Bervillé, ed), 19-24

Faivre-Rampant $P$, Santoni S, Bervillé A (1989) Préparation de l'ADN total de graines sèches : application pour le contrôle des semences par des sondes moléculaires sur des dépôts en taches. CR Acad Sci Sér III, 308, 95-101

Hansen BM, Marcker KA (1984) DNA sequence and transcription of a DNA minicircle isolated from male-fertile sugar beet mitochondria. Nucleic Acids Res 12, 4747-4756

Kemble RJ, Bedbrook JR (1980) Low molecular weight circular and linear DNA in mitochondria from normal and male sterile Zea mays cytoplasm. Nature 284, 565-566

Leclercq P (1969) Une stérilité mâle cytoplasmique du tournesol. Ann Amélior Plant 19, 99-106

Lefort G (1967) In: Mathématiques pour les Sciences Biologiques et Agronomiques. Armand Colin, Paris, 35-36

Maniatis T, Fritsch EF, Sambrook J (1982) Molecular cloning: a laboratory manual, Colds Spring Harbor Laboratory. Cold Spring Harbor, New York, 89-91, 109-112, 250-251 
Owen FV (1945) Cytoplasmically inherited male sterility in sugar beet (Beta vulgaris L). J Agric Res 71, 423-440

Pérez C, Dujon B, Heizmann P, Bervillé A (1988) Sequence of a mitochondrial plasmid of sunflower (Helianthus annuus $\mathrm{L}$ ) and its relationship to other mitochondrial plasmids. Plant Sci 58, 59-69

Powling A (1981) Species of small DNA molecules found in mitochondria from sugarbeet with normal and male-sterile cytoplasm. Mol 8 Gen Genet 183, 82-84

Santoni S, Faivre-Rampant $P$, Moreau E, Bervillé A (1991) Rapid control of purity for the cytoplasm of male-sterile seed stocks by means of a dot hybridization assay. Mol Cell Probes 5 (sous presse)

Thomas CM (1986) The nucleotide sequence and transcription of minicircular mitochondrial DNA's associated with male fertile and cytoplasmic male sterile lines of sugar beet. Nucleic Acids Res 14, 93539379

Vear F (1988) The cytoplasmic male-sterilities now use for sunflower: origin, characteristics and restoring genes used in hybrid seed production. In: Cytoplasmic genetic variability and cytoplasmic male sterility. Colloques INRA vol 45, (A Bervillé, ed), 6573 